\title{
El Greco en la historia del arte occidental en Japón
}

\author{
El Greco in the History of Western Art in Japan
}

\author{
KEIKO OZAKI \\ Departamento Historia y Teoría del Arte \\ Universidad Autónoma de Madrid \\ keiko.ozaki@estudiante.uam.es
}

Resumen: El Museo de Arte Ohara de Japón conserva La Anunciación del Greco, adquirida en París en 1922. En la era Meiji (1868-1912), los pintores japoneses de estilo occidental pusieron el foco de atención en Francia como modelo pictórico. Paralelamente, la revalorización del Greco comenzó en Francia y fue extendiéndose progresivamente por Europa. En el presente trabajo se trata de mostrar el itinerario histórico en el que los pintores japoneses conocieron al Greco a través de sus contemporáneos franceses, llegando a interpretar las obras del Greco en su propio contexto.

Palabras clave: El Greco, Suda Kunitarō, Shirakaba, Museo de Arte Ohara.

Abstract: The Ohara Museum of Art in Japan preserves The Annunciation by El Greco, which was purchased on 1922 in Paris. During the Meiji era (1868-1912), the Japanese painters with western style focused their attention on France as a pictorical reference. At the same time, the re-evaluation of El Greco began in France and gradually was spread throughout Europa. This paper attempts to show the historical itinerary in which those Japanese painters discovered El Greco through their contemporary French painters and, finally, came to interpret El Greco's works by their own context.

Keywords: El Greco, Suda Kunitarō, Shirakaba, Ohara Museum of Art.

1. El arte español en Japón. Introducción

En Japón resulta complicado tener la oportunidad de contemplar de manera directa obras de arte españolas, especialmente aquellas anteriores al siglo XVIII. De hecho, actualmente solo podemos encontrarlas en la Colección Suma del Museo de Arte de 
la Prefectura de Nagasaki ${ }^{1}$, junto con las obras Santa Catalina de Alejandría de Bartolomé Murillo en el Museo de Arte de la Prefectura de $\mathrm{Mie}^{2}$, La Crucifixión del Greco en el Museo Nacional de Arte Occidental en Tokio ${ }^{3}$ y La Anunciación del Greco en el Museo de Arte Ohara en Kurashiki (fig. 1) ${ }^{4}$.

Casi todas estas obras llegaron a Japón después de la Segunda Guerra Mundial. No obstante, La Anunciación del Greco del Museo de Arte Ohara había sido adquirida con anterioridad, justo después de la Primera Guerra Mundial, en 1922. Antes de la obra del Greco, tan solo dos pinturas españolas habían llegado a Japón: una de Murillo y otra de Goya. La obra de Murillo, Virgen María, llegó a Japón en 1912 gracias a un japonés, pero en el mismo año de su llegada se puso en duda su procedencia y, actualmente, no conocemos su paradero. Por otra parte, la obra de Goya, Retrato de un niño, llegó a Japón como una parte de la colección de Matsukata Kōjirō 松方幸次郎 en 1920 y fue presentada al público poco después. Sin embargo, esta obra se puso a la venta en el Museo Metropolitano de Tokio en 1929 y, de nuevo, desconocemos su paradero actual" ${ }^{5}$. En este contexto, La Anunciación del Greco fue la única obra maestra de "los pintores españoles" en Japón hasta los años sesenta, orientando a los japoneses sobre la idea del arte español.

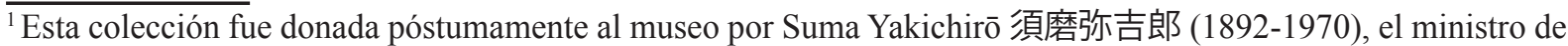
la embajada de Japón en España desde 1940 hasta 1946. Contiene obras del arte español desde el siglo xv hasta la primera mitad del siglo xx. Fukumitsu, Yoko (ed.), The Suma Collection Revisited: 500 Years of Spanish Art, catálogo de la exposición (Nagasaki Prefectural Art Museum, del 23 de abril al 5 de junio de 2005), Nagasaki, Nagasaki Prefectural Art Museum, 2005.

${ }^{2}$ La obra fue adquirida por el museo en 1991. Pérez Sánchez, Alfonso E. y Benito Navarrete Prieto (eds.), El joven Murillo, catálogo de exposición (Museo de Bellas Artes de Bilbao, del 19 de octubre de 2009 al 17 de enero de 2010, Sevilla, Museo de Bellas Artes de Sevilla, del 18 de febrero al 30 de mayo de 2010), Bilbao, Museo de Bellas Artes de Bilbao, 2009.

${ }^{3}$ La Crucifixión del Museo Nacional de Arte Occidental (en adelante MNAO ) perteneció en un primer momento a Bernheim-Jenue en París y, en 1926, pasó a manos del Dr. Hermann Eissler de Viena (aunque Harold Wethey indicó Eiser y el MNAO escribió Eisner, Fernando Marías advirtió que ambas grafías eran erróneas). En 1961 pasó por Wildenstein \& Co en Nueva York. En 1973 fue trasladada a Wildenstein en Tokio y, posteriormente, fue adquirida por MNAO en 1974. Wethey consideró que era una obra del taller del pintor y sugirió la posibilidad de que procediese de la colección de Ramón Díaz de Jerez de la Frontera, mientras tanto el MNAO identificó que el primer propietario de esta obra fue el Duque de Alba. Wethey, Harold, El Greco y su escuela, vol. 2, Madrid, Guadarrama, 1967; Marías, Fernando, "Luces y sombras de una pasión: Zuloaga y el Greco", en Cuadernos de Arte de la Universidad de Granada, 40 (2009), pp. 317-352; Yamada, Chisaburoh F., "Nouvelles acquisitions (catalogue)", en Bulletin Annuel du Musée National d'Art Occidental, 9 (1974), pp. 6-7.

${ }^{4}$ Esta obra tiene casi la misma composición que otras dos Anunciaciones en el Museo de Bellas Artes de Budapest y el Museo de Arte de Toledo (Ohio). Solo en la obra de Ohara la Virgen María tiene la corona de doce estrellas. Wethey explicó que ni el Greco ni su discípulo representaron este tipo de nimbo. Algunos investigadores como Marianne Haraszti-Takzics sospechan que esta obra fue realizada por Jorge Manuel. A pesar de que todavía hay margen para la reconsideración del autor de esta obra, voy a tratarla como obra del Greco dentro de este artículo según la estimación de 1922. En Wethey, Harold, El Greco y su... op. cit., pp. 49-50; The National Museum of Western Art (ed.), El Greco Exhibition, catálogo de la exposición (Tokio, The National Museum of Western Art, del 18 de octubre al 14 de diciembre de 1986; Nara, Nara Prefectural Museum of Art, del 6 de enero al 1 de marzo de 1987; Aichi, Aichi Prefectural Art Gallery, del 11 al 24 de marzo de 1987), Tokio, Tokyo Shimbun, 1986, p. 197. ${ }^{5}$ Sobre la procedencia de las obras de Goya y de Murillo ver Matsuda, Keiji y Tsukasa Machida, "La recepción del arte español en el Japón de las primeras tres décadas del siglo XX", en Cabañas Bravo, Miguel y Wifredo Rincón García (eds.), Imaginarios en conflicto "lo español” en los siglos XIX y XX, Madrid, Consejo Superior de Investigaciones Científicas, 2017, pp. 97-116.
} 
Figura 1: El Greco, La Anunciación, hacia 1600, óleo sobre lienzo, 108,5 x 79,5 cm, Museo de Arte Ohara, Kurashiki.

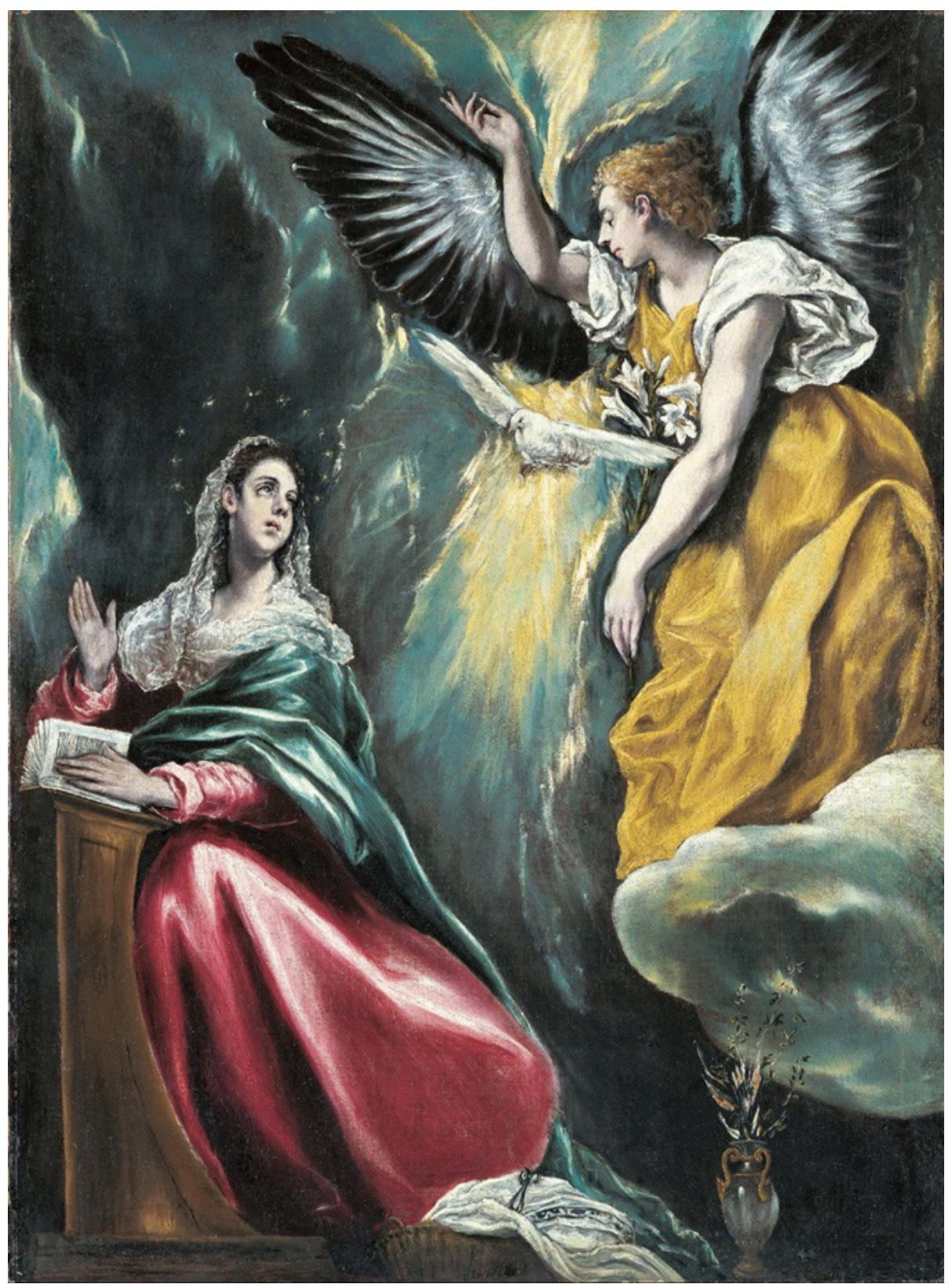

Fuente: Museo de Arte Ohara.

La primera transculturación clara de la obra del Greco en Japón se produjo en 1910. El pintor de estilo occidental Yuasa Ichirō 湯浅一郎 (1868-1931) presentó por primera vez al Greco en la revista Bijutsu shnipō 美術新報 (La nueva noticia del arte), doce años antes de la llegada de La Anunciación. Después de su artículo, algunas personas cultas, sobre todo los pintores japoneses que realizaron su aprendizaje en Europa, empezaron a mostrar interés en el Greco. En la primera mitad del siglo xx, la mayor parte de los pintores de estilo occidental en Japón intentó asimilar la teoría artística a partir de los pintores contemporáneos en Francia. En 
este contexto, la adquisición del Greco fue el resultado del desarrollo de la interpretación del arte español en Francia y el progreso rápido dentro del mundo de arte en Japón.

\section{Transculturación del arte occidental en Japón: desde 1543 hasta 1906}

En este contexto, conviene que nos retrotraigamos al comienzo de la relación entre Japón y Occidente en el siglo XVI. En 1543 los portugueses arribaron por casualidad a una isla del sur de Japón, Tanegashima. En ese momento, Portugal intentaba extender su territorio en Asia, habiendo tomado previamente posesión de Goa (India) en 1510 y Malaca (Malasia) en 1511. Aprovechando esta oportunidad, los barcos comerciales portugueses llegaron a Japón en 1550. A España, el país que se disputó con Portugal la hegemonía del "Nuevo Mundo", le costó tiempo dominar Filipinas y comenzó a comerciar con Japón en 1584ㅎ․

El intercambio comercial entre Japón y ambos países ibéricos se vinculó estrechamente con la evangelización católica. En 1549 el jesuita Francisco Javier llegó a Kagoshima y empezó su misión. Después del éxito de los jesuitas, franciscanos, dominicanos y agustinos empezaron a su vez la evangelización en Japón. Sin embargo, la buena relación entre el gobierno de Japón y la Iglesia Católica duró menos de un siglo. En 1613 Shogunato Tokugawa (16031868) anunció oficialmente la expulsión de los misioneros extranjeros y la prohibición del cristianismo para evitar el aumento del poder de los católicos en Japón ${ }^{7}$. Además, en 1624 rechazó a los barcos españoles y, en 1639, a los portugueses. Esta política supuso la ruptura de las relaciones diplomáticas e interrumpió las relaciones culturales entre Japón y la península ibérica hasta el siglo XIX.

Aunque la relación duró menos de un siglo, la influencia de Portugal y España dio lugar a una nueva producción artística, la laca Namban 南蛮漆器8. Además, los misioneros construyeron seminarios para educar a los cristianos japoneses y en algunos de ellos llegó a enseñarse la técnica de la pintura occidental y del grabado. Las pinturas religiosas realizadas en Europa llegaron entonces a Japón, al mismo tiempo que los cristianos de este país intentaron

\footnotetext{
${ }^{6}$ Ochi, Yūjirō (ed.), Via Orientalis, catálogo de la exposición (Tokio, Sezon Museumof Art, del 8 de abril al 23 de mayo de 1993, Shizuoka, Shizuoka Prefectural Museumof Art del 1 de junio al 4 de julio de 1993, Kioto, The Museum of Kyoto, del 13 de julio al 31 de agosto de 1993, Oita, Oita Prefectural Hall of Arts, del 11 de septiembre al 11 de octubre de 1993), Tokio, 1993.

${ }^{7}$ En 1587 Toyotomi Hideyoshi 豊臣秀吉 (1537-1598) intentó expulsar a los misioneros; sin embargo, solo prohibió el cristianismo y fomentó el comercio con Portugal y España. Por esta razón la prohibición del cristianismo en ese momento resultó en vano: Kobe City Museum, Namban Arts Selection, Kobe, 1998, pp.106-107.

${ }^{8}$ Las lacas Namban son objetos realizados en laca japonesa, denominada laca urushi. La mayor parte de ellos fueron manufacturados para la exportación: Kawamura, Yayoi et al., Laca Namban. Brillo de Japón en Navarra, catálogo de la exposición (Pamplona, Museo de Navarra del 29 de octubre de 2015 al 28 de febrero de 2016), Pamplona, Museo de Navarra, 2016.
} 
realizar pinturas religiosas con estilo occidental. Sin embargo, después de la prohibición del cristianismo estas pinturas de estilo occidental declinaron rápidamente9

Figura 2: Shiba Kōkan, Ryogokubashi (TWEELANDBRUK), 1787, grabado calcográfico, 27,1 x 40,7 cm., Kobe City Museum, Kobe.

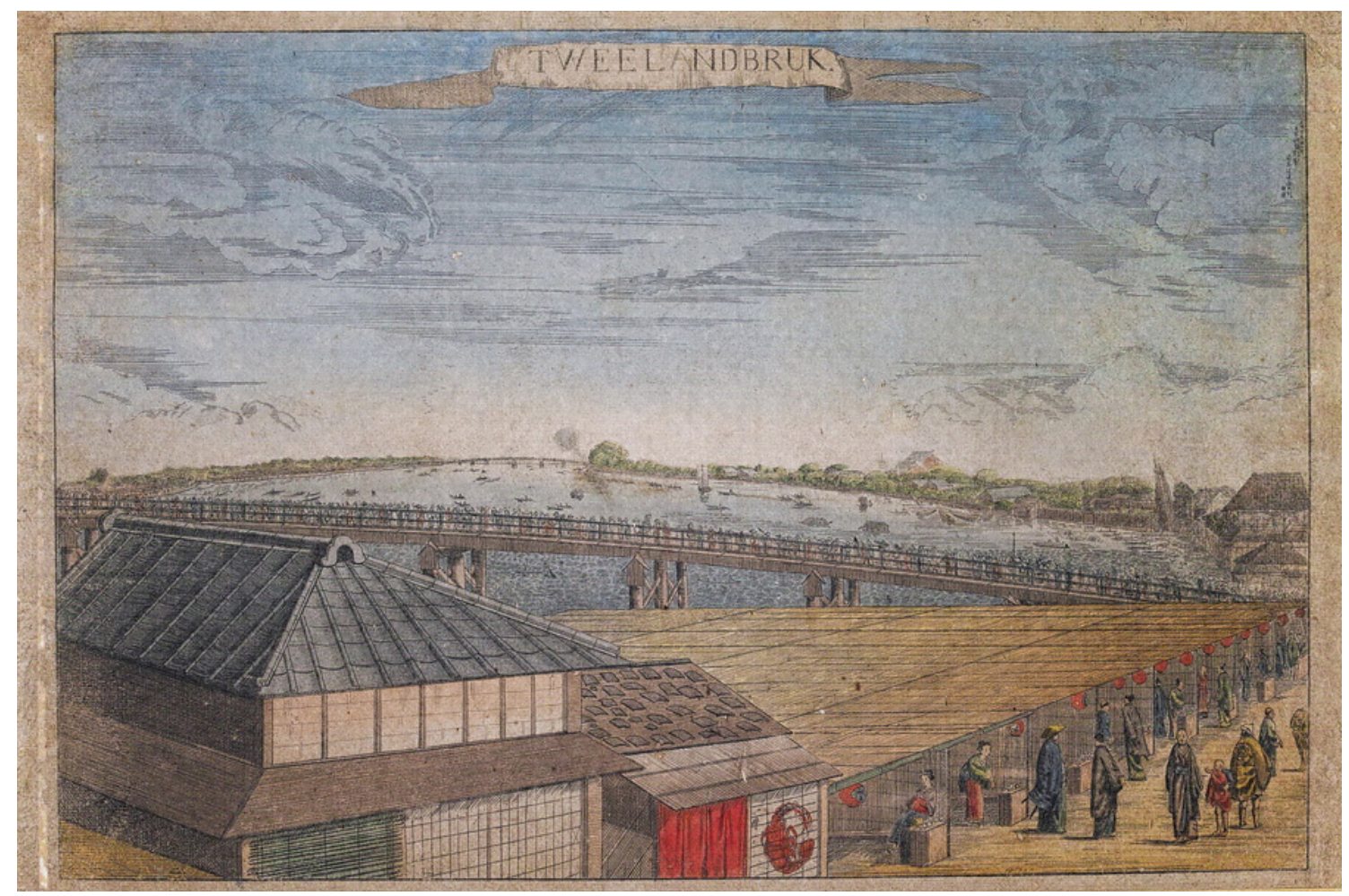

Fuente: Kobe City Museum.

Por otra parte, Holanda empezó a expandirse poco después de que lo hicieran los países ibéricos. Los holandeses llegaron a Japón en 1600 y, en 1609, consiguieron que el shogunato Tokugawa les otorgara el permiso de comercio. Aquel país separó la evangelización del comercio, correspondiendo este último al gobierno japonés. Después del rechazo a Portugal y a España, los barcos holandeses siguieron llegando a una zona limitada de Japón y algunos pintores —como Hiraga Gennai 平賀源内 (1728-1780) o Shiba Kōkan 司馬江漢 (1847$1818)^{10}$ (fig. 2) — comenzaron a aprender la perspectiva o el claroscuro a través de las pinturas y los grabados traídos por los holandeses.

En el siglo XIX, Estados Unidos instó a Japón a que abriese los puertos al extranjero como escala del viaje a través del Pacífico. En 1854 se firmó el Tratado de Kanagawa con

\footnotetext{
${ }^{9}$ Sakamoto, Mitsuru et al., Namban bijutsu to yōfuga (Arte Namban y la pintura de estilo oriental), Genshoku Nihon no bijutsu (Reproducciones de a todo color de arte japonés), vol. 25, Tokio, Shōgaku-kan, 1970, pp. 192196.

${ }^{10}$ El estudio de las pinturas holandesas nació durante la primera mitad del siglo XVIII. El octavo shōgun, Tokugawa Yoshimune 徳川吉宗, permitió importar libros holandeses, con la excepción de aquellos relativos al cristianismo, y fomentó la investigación en campos como la medicina, la astronomía y las ciencias naturales europeas a través del idioma holandés. Las ilustraciones dentro de estos libros tuvieron gran impacto y algunos vasallos empezaron a aprender la pintura desde estos libros: Sakamoto, Mitsuru et al., Namban bijutsu to yōfuga... op. cit., pp. 198-233.
} 
Estados Unidos y en 1858, el Tratado de Amistad y Comercio. En el mismo año, Japón concertó un tratado similar con Holanda, Rusia, Reino Unido y Francia, comenzando así el comercio occidental. En 1868, cuando se derrocó al shogunato Tokugawa, el nuevo gobierno japonés (gobierno Meiji) comenzó el comercio con España. El nuevo gobierno tuvo su mira puesta en el fortalecimiento del país para hacer competencia a las potencias del mundo y fomentó activamente la asimilación del régimen político, el conocimiento y la cultura occidentales. El gobierno invitó a profesores, ingenieros y medios extranjeros a Japón para aprender y difundir su conocimiento. Si consideramos el número de extranjeros por nacionalidades, podemos entender que el nuevo gobierno veía con buenos ojos a países como Reino Unido, Francia, Estados Unidos y Alemania ${ }^{11}$.

Aunque la mayor parte de los extranjeros eran ingleses y además el pionero japonés en el ámbito la pintura de estilo occidental en la época moderna, Takahashi Yuichi 高橋由一 (18281898) $)^{12}$, era discípulo del artista y caricaturista inglés Charles Wirgman, el primer país que los pintores japoneses tomaron como modelo fue Italia. En 1876 abrió sus puertas en Tokio Kōbu Bijutusu Gakkou 工部美術学校, la primera escuela estatal de bellas artes de Japón y el gobierno invitó a arquitectos, escultores y pintores de Italia para educar a los alumnos japoneses ${ }^{13}$. Matsuoka Hisashi 松岡壽 (1864-1892) $)^{14}$, uno de los alumnos de esta escuela, fue a estudiar a Italia en 1880 y se graduó en la Academia di Belle Arti di Roma. Tras regresar a Japón, en 1889 estableció Meiji Bijutusu Kai 明治美術会 (Asociación de Arte Meiji). Matsuoka dedicó su carrera a enseñar a los jóvenes pintores el estilo occidental en diversos institutos de bellas artes.

Por otra parte, en la primera etapa del siglo XIX, los artistas japoneses centraron su atención en Francia como el epicentro de la modernidad. Kuroda Seiki 黒田清輝 (1866-1924) fue a estudiar a Francia en 1884 y se hizo discípulo de Louis Joseph Raphael Collin. Durante sus nueve años en Francia aprendió el estilo impresionista. En 1896 formó un grupo de pintores, Hakubakai 白馬会 (Asociación de Caballo Blanco), y estableció un estilo del plenairismo influido por la escuela impresionista. En el mismo año Tōkyō Bijutsu Gakkō 東京美術学校, la escuela de Bellas Artes de Tokio, creó el departamento de la pintura occidental y Kuroda empezó a enseñar en él. Sus discípulos mejor conocidos fueron Fujishima Takeji 藤島武二 (1867-1943), Okada Saburōsuke 岡田三郎助 (1869-1939) y Wada Eisaku 和田英作 (18761959).

\footnotetext{
${ }^{11}$ Según la investigación de Shoji Uemura, el número total de empleados extranjeros hasta 1890 era de 2690. La mayor parte eran ingleses (1127), estadounidenses (414), franceses (333), chinos (250) y alemanes (215). En consecuencia, casi la mitad de los empleados era ingleses. Uemura, Shoji, "Salaries of Oyatomi (Japan's Foreign Emplyees) in Early Meiji", en Journal of the University of Marketing and Distribution Sciences. Distribution sciences \& business administration, vol. 21, 1 (2008), pp. 1-24.

${ }^{12}$ Takahashi Yuichi nació en la última época del shogunato Tokugawa y aprendió la pintura al óleo en el departamento de arte de Yōsho Sirabesho 洋書調所 (Instituto para el estudio de los libros occidentales) del shogunato. En 1866 se hizo discípulo de Charles Wirgman, que vivía en Yokohama en ese momento.

${ }^{13}$ Kawakami, Mari, "Italia nel campo delle Belle Arti: Hisashi Matsuoka, pittore e KingoTatsuno, architetto (Intellectual encounters with Italy: 2. Italian travels and Aesthetic sense)", en Ritsumeikan studies in language and culture, vol. 20, 2 (2008), pp. 85-99.

${ }^{14} \mathrm{Su}$ maestro era el pintor italiano de paisajes Antonio Fontanero (1818-1882). Fontanero trabajó como profesor en la escuela desde 1876 hasta 1878.
} 
2. El Greco y el arte español en Japón

Al inicio del desarrollo del arte occidental en Japón, los países de referencia para los pintores nipones eran Italia y Francia. Sin embargo, algunos artistas o intelectuales, si bien en menor número, fijaron su mirada en la pintura española al tiempo que se formaban a través del arte italiano o francés.

\subsection{Yuasa Ichirō}

La primera persona que tuvo parte en la divulgación del arte español en Japón fue Yuasa Ichirō. Era discípulo de Kuroda Seiki y Kume Keiichirō 久米桂一郎 (1866-1934) ${ }^{15}$ en la Escuela de Bellas Artes de Tokio. Desde 1950 hasta 1954 estuvo en Europa aprendiendo pintura y permaneció en España durante un año y medio. Cuando se alojó en Madrid, copió seis obras de Velázquez y dos de Murillo en el Museo del Prado. Sus copias incluyeron Marte, Las Meninas, Menipo y Las Hilanderas. Después de regresar a Japón, expuso una obra realizada en París, El paisaje de España, en Nikaten 二科展 —la exposición de Nika Asociación一. Mientras duró su estancia en España, conoció algunos pintores japoneses y se esforzó para que ellos pudiesen copiar las obras de Velázquez. Por ejemplo, el pintor de paisajes Yoshida Hiroshi 吉田博 (1876-1950) realizó un viaje entre 1903 y 1906 en el que visitó Estados Unidos, algunos países europeos, Marruecos y Egipto. En los últimos momentos de este viaje, Yoshida pasó por España y conoció por casualidad a Yuasa. Gracias a su ayuda, Yoshida realizó una copia de Menipo de Velázquez en el Prado ${ }^{16}$. Por otra parte, Yamashita Shintarō 山下新太郎 (1881-1966), el discípulo de Kuroda Seiki, se formó en París desde 1905 y estudió con Louis Joseph Raphael Collin y Fernand Cormon. En 1907 Yamashita viajó a España con el apoyo de Yuasa y copió obras de Velázquez, como La rendición de Breda.

Además de ayudar a los pintores japoneses a aprender el arte español, sobre todo el de Velázquez, Yuasa escribió algunos artículos en las revistas japonesas sobre los pintores en España como el Greco, Ignacio Zuloaga o el propio Velázquez ${ }^{17}$. La actitud de Yuasa, que

\footnotetext{
${ }^{15}$ Kume Keiichirō fue uno de los pintores que estuvieron en París y aprendieron el plenairismo. Permaneció siete años en Francia y en su segundo año en este país (1888) tuvo la oportunidad de visitar España como administrativo del Pabellón Japonés en la Exposición Universal de Barcelona (1888). En España tuvo dos ocasiones para visitar el Museo del Prado. En este museo bosquejó las obras de Rubens, Veronese, Guido Reni, Van Dyck y Murillo. Este pintor centró la atención a las obras de los pintores italianos y flamencos. En el caso de los pintores españoles, no dejó ningún bosquejo de Velázquez pero sí realizó algunos de Murillo: Kawase, Yusuke (ed.), Velázquez and the celebration of painting: the Golden Age in the Museo del Prado, catálogo de la exposición (Tokio, The National Museumof Western Art, del 24 de febrero al 27 de mayo de 2018, Hyogo, Hyogo Prefectural Museum of Art, del 13 de junio al 14 de octubre de 2018), Tokio, The National Museumof Western Art, 2018, pp. 295-298.

${ }^{16}$ Ibidem, p. 296; Kojima, Kaoru, "Fujishima Takeji ni yoru Kuroda Seiki, Kume Keiichirō ate shokan ni tsuite, 3, (Sobre las cartas de Fujishima Takeji a Kuroda Seiki, Kume Keiichirō, 3)", en The Journal of Art Studies, 417 (2016), pp. 78-85.

${ }^{17}$ Takaoka, Chikako, "Japan and El Greco", en NHK Promotion Inc. (ed.), El Greco’s Visual Poetics, catálogo de exposición (Osaka, The National Museum of Art, del 16 de octubre al 24 de diciembre de 2012; Tokio, Tokyo
} 
mostró un gran interés por el arte español, así como el hecho de que se alojara en España durante un periodo largo, era poco habitual para los pintores japoneses contemporáneos.

[...] Por ejemplo, (Yuasa) coleccionó los calzados o los peines, los objetos que normalmente la gente no suele coleccionar. En el caso de su viaje a Occidente, solo Yuasa fue a España e investigó Velázquez, como sabemos, aunque en esa época todo el mundo se dirigió a Francia. En estos días, no es muy raro la gente que investigue el Greco o Velázquez, pero era un punto de genialidad de Yuasa que estudiase Velázquez en aquel momento. ${ }^{18}$

\subsection{Revista Shirakaba 白樺}

Hacia 1910, un grupo de escultores de clase alta con estilo humanista y gusto occidental accedió al mundo literario. Eran Mushanokōji Saneatsu 武者小路実篤 (1885-1976), Arishima Takeo 有島武郎 (1878-1923) y Shiga Naoya 志賀直哉 (1883-1971). Todos ellos publicaron una revista con el nombre de Shirakaba (abedul blanco) y los miembros que presentaron sus obras y críticas en este lugar se denominaron Shirakabaha 白樺派 (la sociedad de Shirakaba). La gran contribución de esta revista fue dar a conocer el arte occidental, sobre todo a Paul Cézanne, Paul Gauguin, Vincent Van Gogh y François Auguste René Rodin. Una costumbre extraordinaria de los miembros de Shirakaba era el intercambio epistolar con los artistas europeos. Aquellos con los que se comunicaron fueron Rodin, Johann Heinrich Vogeler, Max Klinger y Henri Matisse ${ }^{19}$.

Aunque la revista Shirakaba presentó algunos pintores alemanes o ingleses, claramente mostró un gusto hacia los pintores franceses en el campo de arte moderno. Los artistas occidentales a los que se dedicó el número especial de la revista son Van Gogh (en once ocasiones), Cézanne (en nueve) y Rodin (en siete). En contraste, no aparecieron demasiados artistas españoles, aunque sí contamos con algunos casos como el de Goya (en tres ocasiones), el Greco (en una) y Picasso, que aunque no contó con un número especial, su obra fue presentada en una ocasión ${ }^{20}$. En 1914, Koizumi Magane 小泉鐵 (1886-1954) escribió un breve artículo sobre el Greco consultando la investigación de August Liebmann Mayer ${ }^{21}$. Koizumi era traductor y escritor, y su especialidad era el idioma alemán ${ }^{22}$. Aunque no era experto en arte español,

\footnotetext{
Metropolitan Art Museum, del 19 de enero al 7 de abril de 2013), Osaka, The National Museum of Art, 2012, pp. 15-20.

${ }^{18}$ Shirataki, Ikunosuke, "Bōyū Yuasa (Yuasa, difunto amigo)”, en Atelier, vol. 8, 4 (1931).

${ }^{19}$ Kyoto Bunka Hakubutsukan (ed.), "Shirakaba"- pilots of art in modern Japan, catálogo de exposición (Kioto, The Museum of Kyoto, del 6 de junio al 20 de julio de 2009, Tochigi, Utsunomiya Museum of Art, del 26 de junio al 6 de septiembre de 2009, Hiroshima, Hiroshima Museum of Art, del 13 de septiembre al 25 de octubre de 2009 , Kanagawa, The Museum of Modern Art, del 3 de Noviembre al 20 de diciembre de 2009), Kioto, The Museum of Kyoto, 2009, p. 152.

${ }^{20}$ Ibídem, pp. 193-194.

${ }^{21}$ Koizumi, Magane, "El Greco", en Shirakaba, vol. 5, 9 (1914), pp. 169-182.

${ }^{22}$ Sus artículos en la revista Shirakaba son la traducción de Noa, Noa de Gauguin (traducido a su vez de la versión alemana) y la presentación de Klinger, Klimt, Edvard Munch y Rembrandt: Shimizu, Yasutsugu, "Western Art in
} 
tuvo un compañero en Shirakaba, Kojima Kikuo 児島喜久雄 (1887-1950), investigador de la historia del arte occidental, que contaba con El Greco. Siebenfarbige Wiedergabenseiner Gemälde (1913) de August Liebmann Mayer en su biblioteca. Por este hecho, podemos suponer que Kojima asesoró a Koizumi. Por otra parte, Mushanokōji escribió una reseña del artículo de Koizumi y mencionó la influencia que había tenido la pintura del Greco para Julius MeierGraefe $^{23}$. Mushanokōji era uno de los fundadores de Shirakaba y también dominaba el alemán. Por todos estos datos podemos deducir que la mayor parte de las fuentes de la interpretación del Greco en Shirakaba era los investigadores alemanes (figs. 3.1 y 3.2).

Figura 3.1: El Greco, La Sagrada Familia con Santa Ana, detalle, ca. 1595-1596, óleo sobre lienzo, 127 x 106 cm, Hospital de Tavera, Toledo, en Shirakaba, vol. 9, 3 (1918).

Figura 3.2: La portada de Shirakaba vol. 9, 3 (1918).
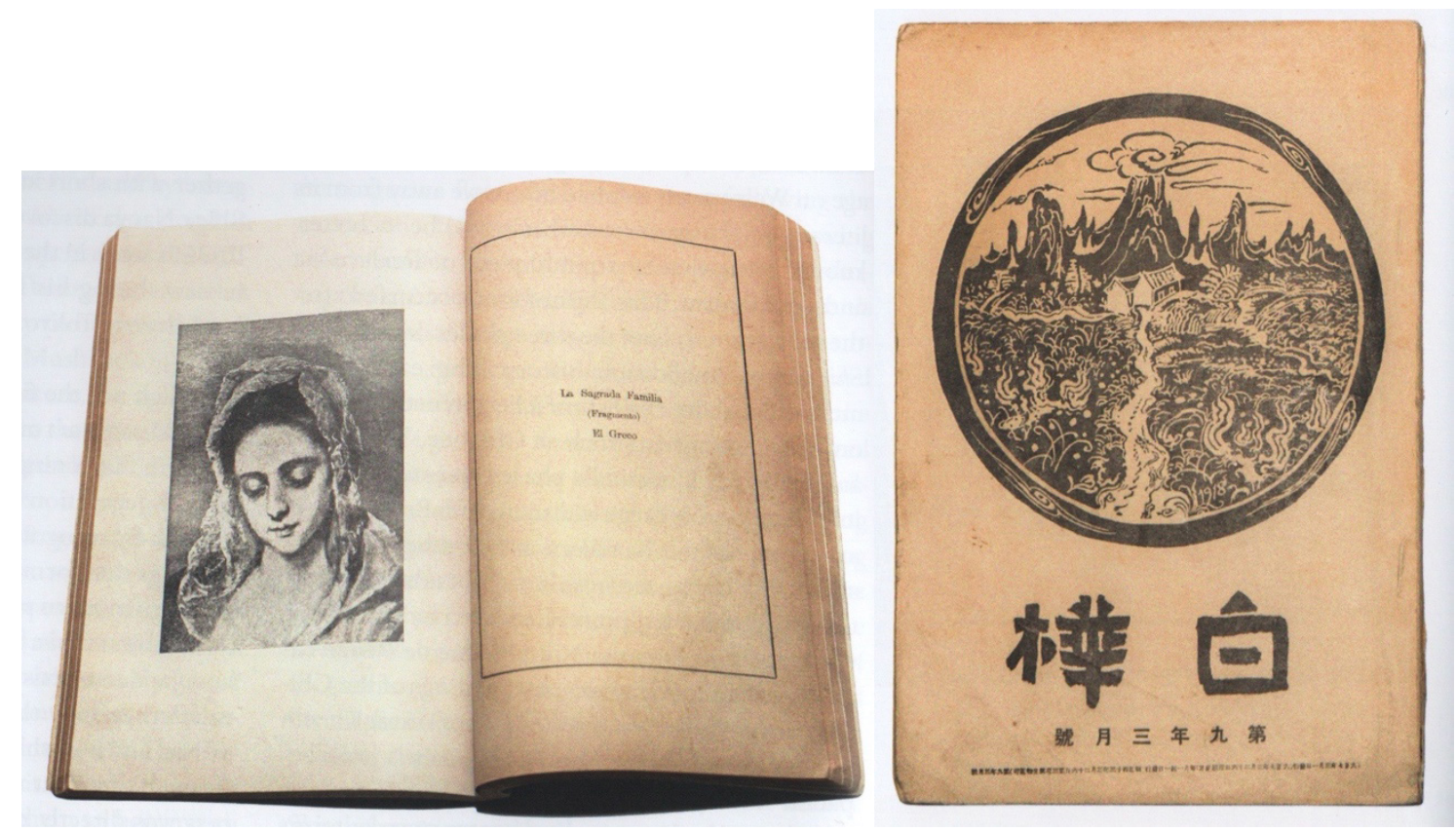

Fuente: Erin Schoneveld, Shirakaba and Japanese Modernism, Leiden, Brill, 2019, pp. 40 y 46.

En 1916 Kimura Shōhachi 木村荘八 (1893-1958), pintor y miembro de Shirakaba, publicó un libro sobre el Greco influido por la investigación de Albert Frederick Calvert y Catherine Gasquoine Hartley ${ }^{24}$. Según este libro, consultó a Hugo Kehrer ${ }^{25}$, Manuel Bartolomé

\footnotetext{
Shirakaba: Focusingon Western Art Introduction in the First Years", en Memoirs of the Graduate School of Letters, Osaka University, 57 (2007), pp. 113-184.

23 “Cuando pensamos sobre Cézanne, inmediatamente recordamos El Greco. Quizá gracias a Meier-Graefe". Reseña en Shirakaba, vol. 5, 9 (1914), p. 183.

24 "Escogí la biografía del pintor según lo que está en El Greco de A. F. Calvert y C. G. Hartley. Además, seguí el orden de los capítulos de este libro". En Shōhachi, Kimura (ed.), El Greco, Tokio, 1916, p. III. El libro de Calvert y Hartley que consultó Kimura era El Greco, An account of his life and works, London, 1909.

${ }^{25}$ Kehrer, Hugo, Die Kunst des Greco, München, 1914.
} 
Cossío y Carl Justi ${ }^{26}$. Por otra parte, también consultó los artículos japoneses de Koizumi y Mushanokōji en Shirakaba y el artículo "Eru Gureko ni tsuite (Sobre el Greco)" escrito por Satō Sōnosuke 佐藤惣之助 (1890-1942) en la revista Ego ${ }^{27}$. Satō era un poeta y su artículo sobre el Greco tuvo una emocionante interpretación.

$[\ldots]$ Cuando veo la obra del Greco, no puedo interpretarlo como solo un pintor
histórico. Era un religioso fuerte lleno de sentimiento puro y sus pinturas
son poder y belleza espiritual que no pudo dejar de pintar cuando recibo la
revelación [...]. Quizá lo que he escrito podrá ser mi ensueño arbitrario, pero
voy a dejar lo que he pensado. ${ }^{28}$

Además, la mayor parte de los miembros de Shirakaba no era investigadores de historia del arte y en la revista se echaba en falta dicha especialidad, aunque intentaron interpretar las obras del Greco consultando los estudios más novedosos de aquel momento. Concedieron mucha importancia a la humanidad de los artistas y podemos entender cómo interpretaron al Greco a través de las palabras de Mushanokoji: "El Greco era uno de los mejores pintores espirituales que más nos gustan"29.

\subsection{Suda Kunitarō 須田国太郎 (1891-1924)}

Suda Kunitarō fue uno de los pintores que excepcionalmente se quedaron en España durante un largo periodo para estudiar el arte español. Al principio estudió historia del arte en la Universidad de Kioto y se especializó en la teoría y la técnica del arte. Por otra parte, aprendió las técnicas de pintura sin ningún maestro. En 1917 ingresó en Kansai Bijutsuin 関西美術院 (Instituto de Arte Kansai) y empezó su aprendizaje como pintor. En 1919 llegó a Madrid y vivió allí hasta 1923. Durante su estancia en España dedicó su tiempo a copiar las obras del Museo del Prado. Al principio, Suda observó la técnica de pintura al óleo y el color de la escuela veneciana. Su principal objetivo en el Prado fue imitar las pinturas italianas del siglo $\mathrm{XVI}^{30}$. En España, Suda copió Venus recreándose en la Música de Tiziano, El rapto de Helena de Tintoretto o La Resurrección del Greco ${ }^{31}$ (fig. 4).

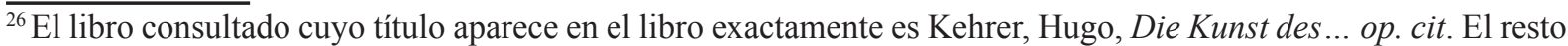
podían haber sido Cossío, Manuel, El Greco, Madrid, Victoriano Suáres, 1908; y Justi, Carl, Diego Velázquez und sein Jahrhundert, Bonn, Cohen, 1888.

${ }^{27}$ Satō, Sōnosuke, "Eru Gureko ni tsuite (Sobre El Greco)”, en Ego, vol. 2, 7 (1914), pp. 107-113.

${ }^{28}$ Ibídem.

${ }^{29}$ La reseña en Shirakaba, vol. 5, 9 (1914).

30 "La razón más grande de mi viaje a España era visitar al Museo del Prado. Es que, por supuesto, para ver varias obras españolas en este lugar. Pero mas que esto, sabía que tiene muchas obras maestras italianas”. En Suda, Kunitarō, "Purado Bijutsukan (el Museo del Prado)", en Bijutsutecho, 25 (1950), pp. 16-17.

${ }^{31}$ Shimada, Yasuhiro y Hidetsugu Yamano (eds.), SUDA Kunitaro: Exhibition, catálogo de exhibición (Kioto, The National Museum of Modern Art, del 1 de noviembre al 18 de diciembre de 2005, Tokio, The National Museum of Modern Art, del 13 de enero al 5 de marzo de 2006, Fukushima, Fukushima Prefectural Museum of Art, del 8 de abril al 14 de mayo de 2006), Kioto, The National Museum of Modern Art, 2005.
} 
Figura 4: Suda Kunitarō, copia de La Resurección de Cristo, del Greco, 1921, óleo sobre lienzo, 209,5 x 96,5cm., Kyoto City Museum, Kioto.

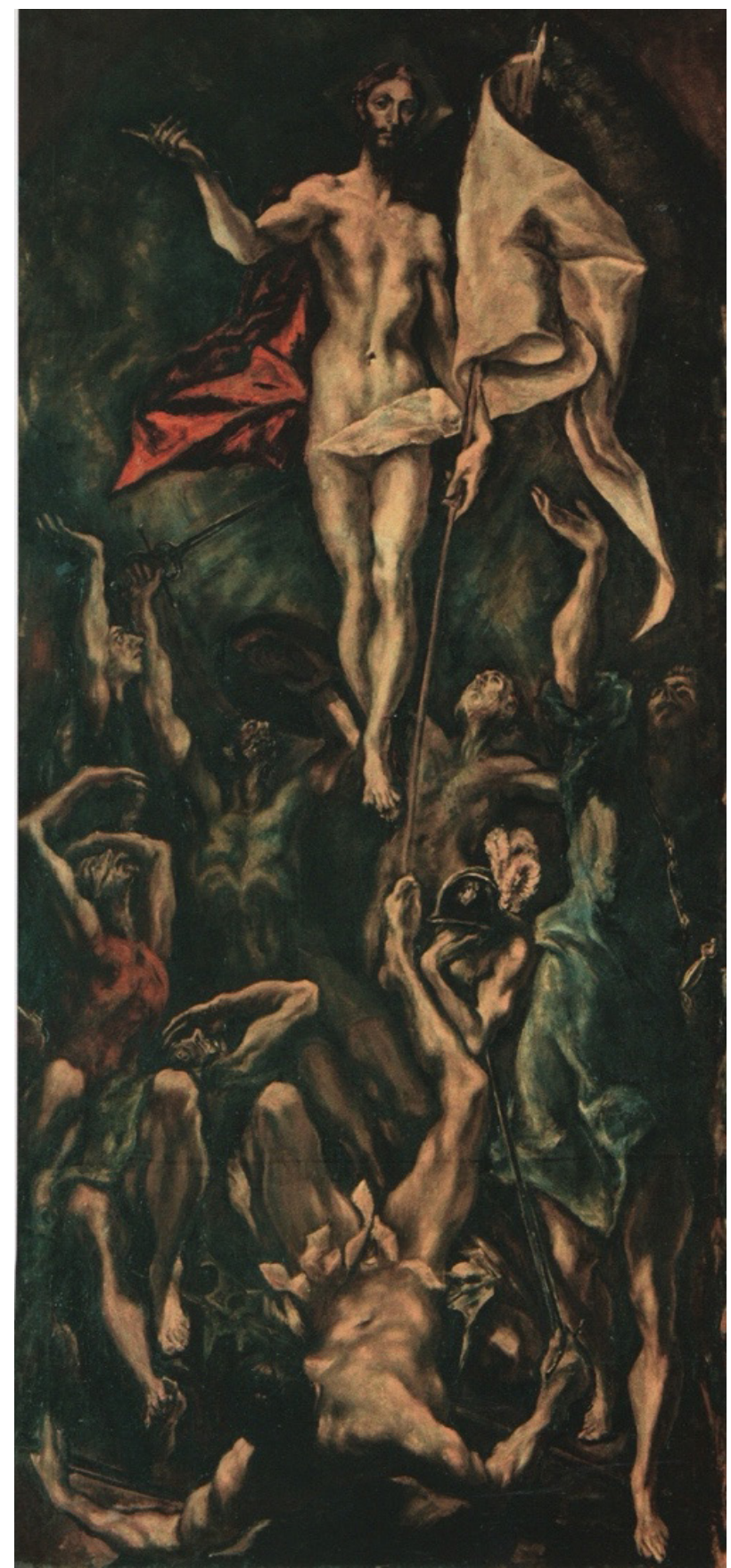

Fuente: Shimada, Yasuhiro y Hidetsugu Yamano (eds.), SUDA Kunitaro... op. cit., p. 44.

Después de su regreso a Japón, Suda publicó muchas investigaciones sobre el arte español como "El color del Greco", "Claroscuro de la pintura barroca", "La maja desnuda y vestida" o "Escuela veneciana" ${ }^{2}$. Suda intentó interpretar al Greco y al arte español, no solo desde el punto de vista de un pintor, sino también del de un investigador. Su interpretación del Greco

$\overline{{ }^{32} \text { Suda, Kunitarō, }}$ Kindaikaiga to rearisumu (La pintura moderna y el realismo), Tokio, 1963. 
era la del creador de la pintura moderna en España y entendió que el color del Greco era algo muy especial.

En España el Greco era la maravilla desde principio. Se dice que Velázquez tuvo obras del Greco y en realidad el color de Velázquez es una referencia directa del Greco. El color de Goya no tuvo la técnica de escuela veneciana, pero tuvo la claridad natural del Greco. Su color es nuevo hoy y será nuevo siempre. $^{33}$

\subsection{Kojima Torajirō 児島虎次郎 (1881-1929) y la colección de Ohara Magosaburō 大原孫三郎 (1880-1943)}

Kojima Torajirō fue un pintor del estilo occidental y discípulo de Kuroda Seiki y Fujishima Takeji. En 1902 fue admitido en la Escuela de Bellas Artes de Tokio y se hizo becario del presidente de hilandería en Okayama, Ohara Magosaburō. Ohara reconoció el talento de Kojima y se convirtió en su mejor mecenas de por vida. Después de su graduación, en 1908 Kojima fue a estudiar a Europa con ayuda de Ohara. Primero se quedó en París y luego estudió en la Escuela de Bellas Artes de Gante. En 1912, tras regresar a Japón, Kojima obtuvo el permiso de Ohara para comprar una obra de Edmond Aman-Jean, con el objetivo de enriquecer el mundo del arte en Japón. Esta obra fue exhibida en Tokio justo después de la vuelta de Kojima y tuvo mucha repercusión ${ }^{34}$. Tras esta experiencia, Kojima sintió la necesidad de llevar más pinturas occidentales a Japón y empezó a adquirir obras europeas con el apoyo de Ohara. Las pinturas adquiridas por Kojima fueron la base para el futuro Museo de Arte Ohara. En 1919, justo después de la Primera Guerra Mundial, Kojima fue a Europa y compró más de 20 obras, incluyendo piezas de Henri Matisse y Monet. En 1921, regresó a Europa para una segunda compra, consiguiendo obras del Greco, Gustave Courbet, Édouard Manet, JeanBaptiste Camille Corot, Edgar Degas, Alfred Sisley y Henri de Toulouse-Lautrec ${ }^{35}$. Kojima fue un pintor que tuvo gran influencia de los impresionistas y, en sus adquisiciones, se observa su gusto por los pintores franceses.

La del Greco es la única obra que representa la España del siglo Xvi y la compra de su pintura fue posible gracias al encuentro con Suda. Durante su primer viaje a Europa, visitó España y conoció a Suda. Ambos viajaron juntos por España y, en 1920, hicieron un viaje en el que recorrieron Bélgica y Holanda. En su diario del día 23 de diciembre de 1919 escribió: "El Museo de la casa del Greco era lo más impresionante"36. También Suda escribió lo siguiente:

\footnotetext{
${ }^{33}$ Ibídem, pp. 174-185.

${ }^{34}$ Sanyo Shinbunsha (ed.), Yume kakeru: Ohara bijutsukan no kiseki (La historia del Museo de Arte Ohara), Okayama, San'yō Shinbunsha, 1991, pp. 316-326.

${ }^{35}$ Yanagisawa, Hideyuki, "Ohara Bijutsikan: Sono Rekishito Gensai (El Museo de Arte Ohara: Su historia y actualidad)", en Journal of Ohara Institute for Social Research, 606 (2009), pp. 22-31.

${ }^{36}$ Sanyo Shinbunsha (ed.), Yume kakeru: Ohara... op. cit., p. 42.
} 
“[...] es la Anunciación del Greco. Esta obra siempre estuvo en la entrada de la galería de Bernheim-Jeune y era muy familiar para mi. El señor Kojima la consiguió fácilmente tan pronto como se volvió del viaje a España"37.

Gracias a la influencia de Suda, en 1922 Kojima compró la obra del Greco por 175000 francos. Esta pintura se convirtió en la obra más importante para la interpretación de la historia del arte español en Japón. Después de la muerte de Ohara, en 1930, se construyó el Museo de Arte Ohara para exponer la colección conseguida por Kojima, en el que aún se encuentra La Anunciación del Greco.

El impacto del Greco en Japón ha sido tratado por intelectuales como Kimura Shōhachi, Koizumi Magane o Suda Kunitarō. En estos últimos años, el estudio más concreto sobre este tema es "Japan and El Greco" de Chikako Takaoka, publicado en 2012 ${ }^{38}$. En este trabajo, se menciona la influencia del libro El Greco o El secreto de Toledo, de Maurice Barrès, publicado en 1911 en París. Kuroda Jūtarō 黒田 重太郎 (1887-1970) ${ }^{39}$ realizó unas traducciones fragmentarias de Barrès al japonés. Barrès puso un especial énfasis en el aspecto místico y espiritual del Greco y la interpretación de este autor francés influyó en investigadores japoneses como Nakai Sotaro 中井 宗太郎 (1879-1966), historiador del arte. Por otra parte, el estudio de Takaoka nos muestra una progresiva aceptación del Greco entre los pintores nipones: parece que en Japón no existían muchos artistas que intentaran aprender el estilo del Greco, aunque se emocionaron con las obras de este pintor. Solo algunas obras, como Ichishudoso no zou 一修道僧の像 (Retrato de un monje) por Kuroda en 1922 (colección privada) o Zugaikotsuo moterujigazou 頭蓋骨を持てる自画像 (Autorretrato con la calavera) por Nakamura Tsune 中村彝 en 1923 (Museo de Arte Ohara), insinúan la influencia indirecta del Greco. La actitud de los pintores japoneses hacia el Greco es muy distinta de la actitud hacia los pintores franceses, cuya técnica intentaron introducir activamente. Esta diferencia podría ser clave para entender la interpretación del arte español en Japón.

\section{La revalorización del Greco en Europa}

Como hemos visto, entre 1906 y 1922, la mayor parte de los pintores japoneses se dirigió a París para conocer la técnica y la teoría del arte, y algunos se vieron influenciados por el arte español. En ese momento es evidente el impacto de Francia y parte de Alemania en el desarrollo de la historia del arte español en Japón pero, sobre todo, en la valoración del Greco.

\footnotetext{
${ }^{37}$ Suda, Kunitarō, "Oharakorekushon ni tsuite (Sobre la colección de Ohara)”, en Bijutsutecho, 80 (1954), pp. 9-10.

${ }^{38}$ Takaoka, Chikako, "Japan and El Greco"... op. cit, pp. 15-20.

${ }^{39}$ Pintor del estilo occidental que visitó París en dos ocasiones, 1918 y 1921, viéndose influenciado por el cubismo francés y por autores como André Lhote. Establecido en París, viajó a Toledo en 1922.
} 
El pintor candiota falleció en 1614 en Toledo. Francisco Pacheco, quien lo conoció, escribió que fue un gran filósofo de agudos dichos ${ }^{40}$. Sus contemporáneos, como Alonso de Villegas o Francisco de Pisa, tuvieron palabras positivas sobre las obras del Greco ${ }^{41}$. Jusepe Martínez utilizó los términos "extravagante" y "caprichoso" para definir el arte del Greco en sus Discursos del nobilísimo arte de la pintura, escrito en 1675, pero, aún así, prevaleció la valoración positiva ${ }^{42}$. Sin embargo, este tipo de opiniones desapareció durante el siglo XVII, ya que en el siglo XVIII aquellos que escribieron sobre historia del arte tuvieron una estimación negativa del Greco, tal y como refleja Antonio Palomino en su libro El museo pictórico y escala óptica (1715-1724) ${ }^{43}$. Ceán Bermúdez empleó las palabras de Palomino en Diccionario histórico de los más ilustres profesores de las bellas artes en España (1800) ${ }^{44}$, si bien en sus últimos escritos se aprecia una progresiva valoración de este pintor ${ }^{45}$. La opinión negativa sobre el Greco se asentó durante el siglo XVIII, si bien pintores españoles como Vicente López, José Aparicio, José de Madrazo, Juan Antonio de Ribera o Mariano Fortuny siguieron apreciando el arte del Greco en los siglos XVIII y XIX ${ }^{46}$. Además, el descubrimiento que se hizo de este pintor fuera de España atrajo el interés no solo de Japón, sino también de otros países europeos.

Un gran momento en el redescubrimiento del Greco tuvo lugar durante la invasión del ejército napoleónico, entre 1802 y 1812, con sus saqueos en España. Asimismo, la Desamortización de Mendizábal de 1836 abrió la puerta a la salida de gran cantidad de arte español hacia Francia ${ }^{47}$. Muchas obras de arte españolas fueron recogidas en el Museo del Louvre y, entre 1838 y 1848, se construyó la Galería Española en este museo por orden de Luis Felipe I de Francia ${ }^{48}$, donde las obras del Greco fueron bien valoradas ${ }^{49}$. En ese sentido, aunque la galería se cerró en 1848 y la mayor parte de la obra fue vendida en Londres en 1853, el arte español se difundió dentro de Europa.

\footnotetext{
${ }^{40}$ Pacheco, Francisco, El Arte de la Pintura, Madrid, Cátedra, 2001 (ca.1638), p. 537.

${ }^{41}$ Pisa, Francisco de, Apuntamientos para la II parte de la "Descripción de la imperial ciudad de Toledo", Toledo, Instituto Provincial de Investigaciones y Estudios Toledanos, 1976 ( $1^{\mathrm{a}}$ ed. 1605); Villegas, Alonso de, Flos Sanctorvm: tercera parte: y historia general, en que se escriven las vidas de santos extravagantes, y de varones ilustres en virtud, Madrid, 1674 ( $1^{\text {a }}$ ed. 1588).

${ }^{42}$ Martínez, Jusepe (notas por Julian Gallego), Discursos practicables del nobilísimo arte de la pintura, Madrid, Akal, 1988, pp. 270-271.

${ }^{4342}$ Palomino, Antonio, El museo pictorico y escala óptica, Madrid, Aguilar, 1947, p. 841.

${ }^{44}$ Ceán Bermúdez, Juan Agustín, Diccionario histórico de los más ilustres profesores de las bellas artes en España, tomo V, Madrid, 1800.

${ }^{45}$ Cera Brea, Miriam, "El Greco desde la Ilustración: la mirada de Ceán Bermúdez”, en Almarcha Núñez-Herrador, María Esther et al. (eds.), El Greco El Greco en su IV Centenario: patrimonio hispánico y diálogo intercultural, Cuenca, Universidad de Castilla-La Mancha, 2016, pp. 1295-1308.

${ }^{46}$ Barón Thaidigsmann, Francisco Javier, "La influencia del Greco en la pintura moderna, del siglo XIX a la difusión del cubismo", en Barón Thaidigsmann, Francisco Javier (coord.), El Greco y la pintura moderna, catálogo de exposición (Madrid, Museo Nacional del Prado, del 24 de junio al 5 de octubre de 2014), Madrid, Museo Nacional del Prado, 2014, pp. 101-197.

${ }^{47}$ Martín González, Juan José, "Problemática de la Desamortización en el arte español”, en El Arte de siglo XIX: II Congreso Español de Historia del Arte,11-14 de diciembre de 1978, vol. 1, Valladolid, Comité Español de Historia del Arte, 1978, pp. 23-33.

${ }^{48}$ Buces Aguado, José Antonio, “Un Zurbarán de la colección de Luis Felipe, rey de Francia, en la iglesia de Santa Bárbara de Madrid", en Bienes Culturales, 6 (2006), pp. 135-138.

${ }^{49}$ Gutiérrez Marcos, María del Rosario, "Recorrido por las fuentes historiográficas rehabilitadoras de la figura del Greco", en Alcántara, 70 (2009), p. 28.
} 
Por otra parte, en el siglo XIX se dio la tendencia entre los intelectuales, sobre todo franceses e ingleses, de viajar por curiosidad y búsqueda de lo desconocido y de lo pintoresco. España fue el destino ideal para este tipo de viajes. Como consecuencia, en la segunda mitad del siglo XIX se publicaron muchos libros acerca de los viajes a España, entre los que destacan los de Richard Ford y Théophile Gautier ${ }^{50}$. Aunque el principal objetivo de estos viajes era la búsqueda de lo exótico, según la idea del romanticismo, el interés hacia España impulsó la investigación del arte español fuera del país. Además, el Greco empezó a tener una revaloración entre los artistas que buscaban nueva inspiración. Los románticos, como Théophile Gautier, Gustave Doré y el Barón Davillier, empezaron a considerar al Greco como anti-academicismo y anti-clasicismo ${ }^{51}$. Esta interpretación fue aceptada por pintores como Eugène Delacroix, Jean-François Millet y Manet, quienes se inspiraron en el Greco ${ }^{52}$.

Desde 1870, los investigadores de la historia del arte empezaron a poner en valor al pintor, y autores como Paul Lafort o Carl Justi presentaron estudios sistemáticos sobre el Greco. A pesar de que, en un primer momento, los pintores japoneses recibieron la influencia del redescubrimiento del Greco desde Francia, los investigadores españoles, como Elías Tormo o Francisco Navarro Ledesma, intentaron aclarar hechos históricos sobre este pintor. Sobre todo, Manuel Bartolomé Cossío empezó a investigar sobre el Greco desde 1886 y realizó una primera exposición de su obra en 1902. En 1908 publicó el primer catálogo de las obras del Greco. En este libro, Cossío presentó al pintor como un místico que personificaba la idea de misticismo en la España del siglo XVI, así como el espíritu español ${ }^{53}$. Sin embargo, el Greco no pudo escapar del contexto del arte moderno, ya que desde el siglo XIX hasta la primera mitad del siglo XX las investigaciones sobre este pintor avanzaron a marchas forzadas. En este sentido, podemos recordar a Paul Lafort o Maurice Barrès en Francia, a Meier-Graefe, August Liebmann Mayer y Hugo Kehrer en Alemania o a Roger Fry en Reino Unido. También Max Dvořák representó al Greco como un pintor del manierismo y un místico en su libro publicado en $1921^{54}$. Aunque la estimación negativa se había fortalecido en el siglo XVIII, el Greco seguía siendo uno de los pintores más representativos del siglo XVI en España. Sin embargo, la investigación del Greco que más influyó a los pintores japoneses fue la revalorización realizada en Francia o Alemania, sobre todo la de los pintores románticos.

A pesar de que la interpretación del Greco como un pintor místico tuvo buena acogida entre los investigadores en la primera mitad del siglo xx, en los años ochenta tuvieron lugar dos descubrimientos definitivos: los manuscritos encontrados en el margen de dos libros, Las vidas

\footnotetext{
${ }^{50}$ Sanz Serrano, María Jesús, "Viaje de franceses e ingleses por España en la segunda mitad del siglo XIX. Su aportación a la historia del arte", en El arte del siglo XIX: II Congreso Nacional de Historia del Arte. Valladolid, 11-14 de diciembre de 1978, vol.1, Valladolid, Comité Español de Historia del Arte, 1978, pp. 65-68.

${ }^{51}$ García Rodríguez, Fernando y María Victoria Gómez Alfeo, "La valoración del Greco por los críticos del '98”, en Anales de la Historia del Arte, 12 (2002), pp. 199-225.

${ }^{52}$ Barón Thaidigsmann, Francisco Javier, "La influencia del Greco... op. cit., pp. 16-17.

${ }^{53}$ Rozalén Medina, José Luis, "Manuel B. Cossío: Un siglo del Greco", en Catedra Nova, 28 (2009), pp. 167-184.

${ }^{54}$ Dvořák, Max, The history of art as the history of ideas, London, Routledge \& Kegan Paul, 1984, pp. 105-108.
} 
de los más excelentes pintores, escultores y arquitectos de Vasari y Diez libros de arquitectura de Vitruvio. Los manuscritos fueron traducidos y publicados por Xavier de Salas, Fernando Marías y Agustín Bustamante ${ }^{55}$. Gracias a sus trabajos, el Greco fue valorado como un artista que tuvo la conciencia de expresión del color de Tiziano y de la descripción del cuerpo humano de Michelangelo ${ }^{56}$. De este modo, podemos interpretar que el Greco fue un pintor influido no solo por el misticismo español sino también por artistas italianos, sobre todo Tiziano, y que representó su obra de acuerdo a su idea de la descripción de lo natural.

\section{Conclusión}

La revalorización del Greco que arrancó desde Francia se expandió rápidamente dentro de Europa. Casualmente, en ese momento, los pintores japoneses viajaron a Europa, sobre todo a Francia. Los intelectuales nipones intentaron asimilar la teoría del arte más moderno de Europa e imitaron ávidamente la técnica de los pintores franceses. En este contexto, es indudable que los japoneses fueron conscientes de la creciente atención que se estaba dando a la obra del Greco en Europa. Después de la exposición del Museo del Prado, se publicó el primer catálogo del Greco en 1908, tuvo lugar la exposición en la Real Academia de Bellas Artes de San Fernando en 1909 y se inauguró el Museo del Greco en Toledo en $1911^{57}$.

En Japón, los miembros de Shirakaba estudiaron las obras del Greco a través de los investigadores alemanes. Aunque sus trabajos supusieron una gran contribución para la divulgación del arte occidental en ese país, prefirieron los mitos de los genios a la interpretación más rigurosa desde la óptica histórico-artística. Para ellos, la interpretación del Greco, que comenzó por los pintores franceses y a los que sucedieron los investigadores alemanes, era ideal: la historia de un artista que tuvo un espíritu moderno y que fue rescatado de la ignorancia anticuada, coincidiendo con la figura de "artista occidental" para los Shirakabaha.

Aunque la facción dominante de la interpretación del Greco se adhirió al gusto romántico, algunos investigadores o pintores intentaron analizarlo desde un punto de vista estético o desde la historia del arte. Por ejemplo, dentro de la colección de los libros de Suda, existen no solo investigaciones de los alemanes, sino también estudios sobre el Greco de Cossío, Francisco

\footnotetext{
${ }^{55}$ Salas, Xavier de, Cuatro obras maestras: Vicent Macip, El Greco, Van Dyck, Goya, Madrid, Sociedad de Estudios y Publicaciones, 1966; "Un examplaire des Vies de Vasari annoté par Le Greco", en Gazette des Beaux-Arts, serie 6, 69 (1967), pp. 177-180; Miguel Ángel y El Greco, Madrid, Real Academia de Bellas Artes de San Fernando, 1967; "Las notas del Greco a la Vida de Tiziano de Vasari”, en El Greco: Italy and Spain, Studies in the History of Art, Washington, National Gallery of Art, 1984; Marías, Fernando y Agustín Bustamante, Las ideas artísticas del Greco, Madrid, 1981.

${ }^{56}$ Salas, Xavier de, Miguel Angel y... op. cit. .

${ }^{57}$ Beruete, Aureliano de, Catálogo del Museo del Greco en Toledo, Madrid, 1912.
} 
de Borja San Román (1910) o José Ramón Mélida (1915). Además, Koike Masao tuvo una entrevista con Cossío para pedirle permiso para traducir al Greco al japonés ${ }^{58}$.

Las obras del Greco empezaron a aparecer en el mercado europeo con frecuencia desde 1900. A pesar de que la adquisición de la obra del Greco en 1922 pudo ser fortuita, parece ser el resultado del intento de los japoneses de asimilar el arte occidental. Su obra impresionó muy notablemente a los japoneses, que solo habían visto sus pinturas en las fotos en blanco y negro de los catálogos. Indudablemente, La Anunciación del Museo de Arte Ohara orientó la interpretación del arte español en Japón.

Después de la Segunda Guerra Mundial, en el Museo Nacional de Arte Occidental se celebró una exposición dedicada al Greco, entre 1986 y $1987^{59}$, aunque la exposición de Goya se habría celebrado en 1971-1972 ${ }^{60}$ y la de Velázquez, en $1980^{61}$. Después, entre 2012 y 2013 , se organizó la exposición El Greco ’s Visual Poetics para celebrar el cuarto centenario de su muerte, bajo la supervisión de Fernando Marías, Yoji Yukiyama y Yusuke Kawase ${ }^{62}$. Ha pasado casi un siglo desde la llegada de La Anunciación a Japón y, desde entonces, nuestra interpretación sobre el Greco ha vivido un progresivo desarrollo.

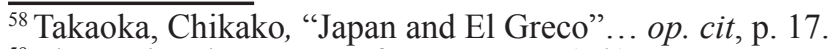

${ }^{59}$ The National Museum of Western Art (ed.), op. cit.

${ }^{60}$ Goya ten kataroguiinkai (Comité de la exposición de El arte de Goya) (ed.), El arte de Goya, catálogo de la exposición (Tokio, The National Museum of Western Art, del 16 de noviembre de 1971 al 23 de enero de 1972 , Kioto, Kyoto Municipal Museum of Art, del 29 de enero al 15 de marzo de 1972), Tokio, The National Museum of Western Art, 1971.

${ }^{61}$ Bunkacho (Agencia para Asuntos Culturales) (ed.), Velázquez y la Pintura Española de su Tiempo, catálogo de la exposición (Tokio, The National Museum of Western Art, del 29 de octubre al 21 de diciembre de 1980), Tokio, The National Museum of Western Art, 1980.

${ }^{62}$ NHK Promotion Inc. (ed.), El Greco's Visual... op. cit.
} 